\begin{tabular}{|c|l|}
\hline Title & Wave front images of acoustic waves in the (100) and (001) surfaces of Te02 \\
\hline Author(s) & Tanaka, Y ukihiro; Takigahira, Masato; Tamura, Shin-ichiro \\
\hline Citation & $\begin{array}{l}\text { PHY SICAL REVIEW B, 66,075409 } \\
\text { https://doi.org/L0.1103/PhysRevB.66.075409 }\end{array}$ \\
\hline Issue Date & 2002 \\
\hline Doc URL & http://hdl.handle.net/2115/5850 \\
\hline Rights & Copyright $\odot 2002$ A merican Physical Society \\
\hline Type & article \\
\hline File Information & PRB66-7.pdf \\
\hline
\end{tabular}

Instructions for use 


\title{
Wave-front images of acoustic waves in the (100) and (001) surfaces of $\mathrm{TeO}_{2}$
}

\author{
Yukihiro Tanaka, Masato Takigahira, and Shin-ichiro Tamura \\ Department of Applied Physics, Hokkaido University, Sapporo 060-8628, Japan
}

(Received 25 February 2002; published 15 August 2002)

\begin{abstract}
Based on the finite-difference time-domain (FDTD) method, we study theoretically the wave-front images of acoustic waves propagating on the (100) and (001) surface of a highly anisotropic tetragonal $\mathrm{TeO}_{2}$ crystal for which imaging experiments have recently been conducted. The theoretical images well reproduce characteristic features observed experimentally. The group-velocity calculations of both surface and pseudosurface acoustic waves account for the shapes and locations of the major wave fronts obtained with the FDTD method. Additional weak wave-front structures that disappear rather quickly in time are attributed to bulk acoustic waves.
\end{abstract}

DOI: 10.1103/PhysRevB.66.075409

PACS number(s): 68.35.Iv, 62.65.+k, 68.35.Ja

\section{INTRODUCTION}

A $\mathrm{TeO}_{2}$ crystal of tetragonal symmetry is known to possess a large elastic anisotropy. The shapes of the calculated bulk slowness (the inverse of bulk sound velocity) are deformed dramatically from circles especially in the (001) plane ( $X Y$ plane) with fourfold symmetry. ${ }^{1}$ In addition, along certain directions the longitudinal sound velocity is slower than the sound velocity of the one of the shear modes. Thus we can expect that this large anisotropy should also affect significantly the acoustic wave propagation on a free surface of the $\mathrm{TeO}_{2}$ crystal. In particular, the shapes of wave front governed by the group-velocity at the surface are expected to exhibit some interesting properties.

Recently, a real-time imaging experiment of acoustic waves at the surfaces of both isotropic and anisotropic materials (including a $\mathrm{TeO}_{2}$ crystal) has been done by Wright and his coworkers. ${ }^{2,3}$ They used the optical pump and probe technique $^{4}$ to periodically excite surface phonons, illuminating an opaque thin film on a transparent substrate. With this technique they have imaged the wave fronts of acoustic waves on surfaces at frequencies up to $1 \mathrm{GHz}$. In addition to the anisotropic shapes of the wave fronts, the observed images show a large variation in acoustic amplitude with respect to the propagation direction. The latter effect is related to the phonon focusing effect ${ }^{5,6}$ in the finite-wavelength regime. $^{7-9}$

The purpose of the present work is to develop in detail the calculation of wave-front images of acoustic waves (both bulk and surface in nature) on the (100) plane ( $Y Z$ plane) with twofold symmetry and on the (001) plane ( $X Y$ plane) with fourfold symmetry for the tetragonal crystal $\mathrm{TeO}_{2}$. This is carried out by solving the elastic wave equations in a crystal with a localized lattice displacement as an initial condition. The finite-difference time-domain (FDTD) method is used to solve the equations numerically. ${ }^{10}$ The FDTD method is a popular numerical scheme for the solution of many problems in wave propagations. It is especially effective for a large-scale simulation of a finite complex system, and has recently been applied to the study of the transmission and frequency spectra of both electromagnetic and acoustic waves in periodic, composite structures called photonic ${ }^{11,12}$ and phononic crystals. ${ }^{13,14}$ The calculated wave fronts should consist of both bulk and surface acoustic modes. We study the time evolutions of these wave fronts and analyze their main features based on the group velocities of both the surface and pseudosurface acoustic modes calculated separately. The comparison with the results of imaging experiments ${ }^{2,3}$ is also made.

\section{FORMULATION}

We study a semi-infinite $\mathrm{TeO}_{2}$ crystal that occupies the half-space $z>0$ with a flat surface at $z=0$ parallel to $x y$ plane. On surfaces, in general, the propagation of bulk, surface and pseudosurface acoustic waves can be observed. Here we briefly recapitulate the formulations we need for obtaining the solutions for those three kinds of acoustic waves. In the present calculation we ignore small piezoelectric effects of $\mathrm{TeO}_{2}$ crystals.

\section{A. Bulk acoustic waves}

The equations governing the elastic wave propagation in crystalline solids are

$$
\rho \ddot{u}_{i}=c_{i j m n} \frac{\partial^{2} u_{m}}{\partial x_{j} \partial x_{n}} \quad(i=1,2,3),
$$

where $\rho$ is mass density of the medium, $\mathbf{u}(\mathbf{r}, t)$ is the lattice displacement vector, $c_{i j m n}$ is the elastic stiffness tensor, and $\mathbf{r}=(x, y, z)=\left(x_{1}, x_{2}, x_{3}\right)$ is a position vector. The summation convention over repeated indices has been assumed. By putting

$$
\mathbf{u}=a \mathbf{e} \exp \{i(\mathbf{k} \cdot \mathbf{r}-\omega t)\},
$$

with $a$ an amplitude, $\mathbf{e}$ a unit polarization vector, $\mathbf{k}$ $=\left(k_{x}, k_{y}, k_{z}\right)=\left(k_{1}, k_{2}, k_{3}\right)$ a wave vector and $\omega$ the angular frequency, Eq. (1) is converted to the eigenvalue equation

$$
\begin{aligned}
\left(M_{i m}-\rho \omega^{2} \delta_{i m}\right) e_{m}= & \left(c_{i j m n} k_{j} k_{n}-\rho \omega^{2} \delta_{i m}\right) e_{m}=0 \\
& (i=1,2,3) .
\end{aligned}
$$

The explicit expression for the matrix $M_{i m}$ for $\mathrm{TeO}_{2}$ crystal (tetragonal, point group 422) is 


$$
M=\left(\begin{array}{ccc}
c_{11} k_{x}^{2}+c_{66} k_{y}^{2}+c_{44} k_{z}^{2} & \tilde{c}_{12} k_{x} k_{y} & \tilde{c}_{13} k_{z} k_{x} \\
\tilde{c}_{12} k_{x} k_{y} & c_{66} k_{x}^{2}+c_{11} k_{y}^{2}+c_{44} k_{z}^{2} & \tilde{c}_{13} k_{y} k_{z} \\
\tilde{c}_{13} k_{z} k_{x} & \tilde{c}_{13} k_{y} k_{z} & c_{44}\left(k_{x}^{2}+k_{y}^{2}\right)+c_{33} k_{z}^{2}
\end{array}\right)
$$

with $c_{I J}$ being the conventional elastic constants with two indices, $\tilde{c}_{12}=c_{12}+c_{66}$ and $\tilde{c}_{13}=c_{13}+c_{44}$. From the eigenvalues $\omega=\omega_{l}(l=1,2,3)$ of Eq. (3) we obtain, for a given wave vector $\mathbf{k}$, the phase velocities $v_{l}=\omega_{l} / k$ with $k=|\mathbf{k}|$ and the group velocities $\mathbf{V}_{l}=\nabla_{\mathbf{k}} \omega_{l}$ of three bulk modes, i.e., slow transverse (ST, $l=1)$, fast transverse (FT, $l=2)$, and longitudinal $(L, l=3)$ modes.

\section{B. Surface acoustic waves}

To obtain the surface wave solutions in a semi-infinite solid occupying a half-space, stress-free boundary condition should be imposed. For example, if the surface is the (001) face ( $X Y$ plane) and the medium occupies $z>0$ with the stress-free surface at $z=0$, Eq. (3) should be solved for $\omega$ and $k_{3}=k_{z}$ with given two-dimensional (2D) wave vector $\mathbf{k}_{\|}=\left(k_{x}, k_{y}\right)$ together with the boundary conditions

$$
\left.\sigma_{i 3}\right|_{z=0}=\left.c_{i 3 m n} \frac{\partial u_{m}}{\partial x_{n}}\right|_{z=0}=0 \quad(i=1,2,3) .
$$

In general, the surface wave solution consists of three partial waves (discriminated by $l=1,2,3$ ) with complex wave numbers $k_{3}=k_{3}^{(l)}\left(\operatorname{Im}\left[k_{3}^{(l)}\right]>0, l=1,2,3\right)$ and takes the form

$$
\mathbf{u}=\sum_{l=1}^{3} a^{(l)} \mathbf{e}^{(l)} \exp \left(i k_{3}^{(l)} x_{3}\right) \exp \left\{i\left(\mathbf{k}_{||} \cdot \mathbf{x}_{\|}-\omega t\right)\right\},
$$

where $\mathbf{x}_{\|}=(x, y)$. The phase velocity $v_{S}=\omega_{S} / k_{\|}, \quad\left(k_{\|}\right.$ $\left.=\left|\mathbf{k}_{||}\right|\right)$, and the group velocity $\mathbf{V}_{S}=\nabla_{\mathbf{k}_{\|}} \omega_{S}$ of the surface waves are derived from the eigenfrequency $\omega=\omega_{S}$ of the surface waves, which is determined from Eq. (3) and the following homogeneous equations equivalent to Eq. (5):

$$
c_{i 3 m n} \sum_{l=1}^{3} k_{n}^{(l)} e_{m}^{(l)} a^{(l)}=0 \quad(i=1,2,3) .
$$

A similar analysis should also be done for the case in which the surface is the (100) face of $\mathrm{TeO}_{2}$ (the medium occupies $x>0$ with the stress-free surface at $x=0)$. In this case Eq. (3) should be solved for $\omega$ and $k_{1}=k_{x}\left(\operatorname{Im}\left[k_{1}^{(l)}\right]\right.$ $>0, l=1,2,3)$ with a given two-dimensional wave vector $\mathbf{k}_{\|}=\left(k_{y}, k_{z}\right)$ on the (100) surface, [i.e., $\mathbf{k}=\left(0, \mathbf{k}_{\|}\right)$and also in this case $\mathbf{r}=\left(0, \mathbf{x}_{\|}\right)$with $\left.\mathbf{x}_{\|}=(y, z)\right]$ together with the boundary conditions $\left.\sigma_{i 1}\right|_{x=0}=0(i=1,2,3)$.

\section{Pseudosurface acoustic waves}

On a stress-free flat surface of an anisotropic semi-infinite solid, branches of acoustic waves called pseudosurface waves (PSW's) exist in some restricted directions. ${ }^{15,16}$ The
PSW's behave as surface waves, though they are not true (nondecaying) surface waves, having a quasitransverse bulk wave component with propagation vector tilted down into the solid. However, their amplitudes decay very slowly as they propagate along the surface and hence PSW's are observed experimentally rather like surface waves. ${ }^{15}$

Thus the PSW's are characterized by a complex 2D wave vector along the surface, but the searching procedure for PSW's is quite similar to that for the surface waves described above. More explicitly, we add a small positive imaginary part to $\mathbf{k}_{\|}$by changing $\mathbf{k}_{\|} \rightarrow \mathbf{k}_{\|}^{\prime}=\mathbf{k}_{\|}(1+i \varepsilon)$ with the attenuation coefficient $\varepsilon>0$, and find a set of solutions $\operatorname{Im}\left[k_{3}^{(l)}\right]$ $>0 \quad\left(\operatorname{Im}\left[k_{1}^{(l)}\right]>0\right), \quad(l=1,2), \quad$ and $\operatorname{Im}\left[k_{3}^{(3)}\right]<0 \quad\left(\operatorname{Im}\left[k_{1}^{(3)}\right]\right.$ $<0)$ for the (001) [for the (100)] surface. These solutions correspond to the wave with amplitude growing into the bulk of the medium.

\section{FDTD method}

In this FDTD scheme, Eq. (1) governing the motion of lattice displacement $\mathbf{u}(\mathbf{r}, t)$ is decomposed into a set of two differential equations of the first order with respect to the space variables,

$$
\begin{gathered}
\rho \ddot{u}_{i}(\mathbf{r}, t)=\partial_{j} \sigma_{i j}(\mathbf{r}, t), \\
\sigma_{i j}(\mathbf{r}, t)=c_{i j m n} \partial_{n} u_{m}(\mathbf{r}, t) .
\end{gathered}
$$

For the calculation we impose the stress-free boundary condition on the upper face of the assumed parallelopiped sample and Mur's first-order absorbing boundary conditions $^{10}$ on the other five faces of the sample. The interval of a unit time step for the time evolution and the sizes of the grids in space are chosen so that the convergence criteria may be satisfied. At the initial time $t=0$, the displacement field with a Gaussian profile $f\left(x_{\|}\right)=\tilde{a} \exp \left[-x_{\|}^{2} /\left(\Delta x_{\|}\right)^{2}\right]$ with $x_{\|}=\left|\mathbf{x}_{\|}\right|$and $\tilde{a}$ a constant, is given at the top of the sample (the surface). This is consistent with the experimental situation. $^{2,3}$ More explicitly the initial displacement profile is

$$
\begin{aligned}
u_{i}\left(x_{\|}, t=0\right) & =\delta_{i, I} f\left(x_{\|}\right) \\
& =\delta_{i, I} \int_{0}^{\infty} F\left(k_{\|}\right) J_{0}\left(k_{\|} x_{\|}\right) k_{\|} d k_{\|},
\end{aligned}
$$

where $I=1$ for the (100) surface and $I=3$ for the (001) surface, and $J_{0}(x)$ is a Bessel function. The expression for the displacement profile $F\left(k_{\|}\right)$in wave-number space is again Gaussian, i.e., $F\left(k_{\|}\right)=\tilde{a} \exp \left[-k_{\|}^{2} /\left(\Delta k_{\|}\right)^{2}\right] /\left[2\left(\Delta k_{\|}\right)^{2}\right]$ with $\Delta k_{\|}=2 / \Delta x_{\|}$, which is obtained with the formula known as the Weber integral 


$$
\int_{0}^{\infty} x J_{0}(\beta x) \exp \left(-\alpha^{2} x^{2}\right) d x=\exp \left(-\beta^{2} / 4 \alpha^{2}\right) / 2 \alpha^{2} .
$$

Once these initial and boundary conditions are specified, Eqs. (8) and (9) can be solved numerically for each normal mode by discretizing both the time and space domains. [The explicit expressions for the discretized versions of Eqs. (8) and (9) are given in the Appendix.] The assumed dimensions of the sample are $100 \mu \mathrm{m} \times 100 \mu \mathrm{m} \times 100 \mu \mathrm{m}$. For the FDTD calculation this sample is divided into small cubic cells; the size of each discretized cell is $0.22 \mu \mathrm{m}$ $\times 0.22 \mu \mathrm{m} \times 0.22 \mu \mathrm{m}$. Thus, from the initial displacement fields at the instant $t=0$, their spatial derivatives are determined using simple finite difference formulas. Equations (8) and (9) then give us the time derivative of the displacement field $\mathbf{u}$, which allows us to update $\mathbf{u}(\mathbf{r}, t)$ for a small but positive time increment. In this way the displacement fields $\mathbf{u}\left(\mathbf{r}, t_{i}\right)$ at discretized points on the time axis $t_{i}$ ( $i$ $=1,2, \ldots$, ) are determined for the sampled 3D grid points. For a sufficiently large number of these $\mathbf{u}$ data on the time axis, the displacement fields on the surface can be plotted.

\section{NUMERICAL RESULTS}

We present numerical results for the acoustic wave propagation on the (100) and (001) planes of $\mathrm{TeO}_{2}$ crystal. ${ }^{17}$ The time step used for the FDTD calculations is $0.005 \mathrm{~ns}$ and the

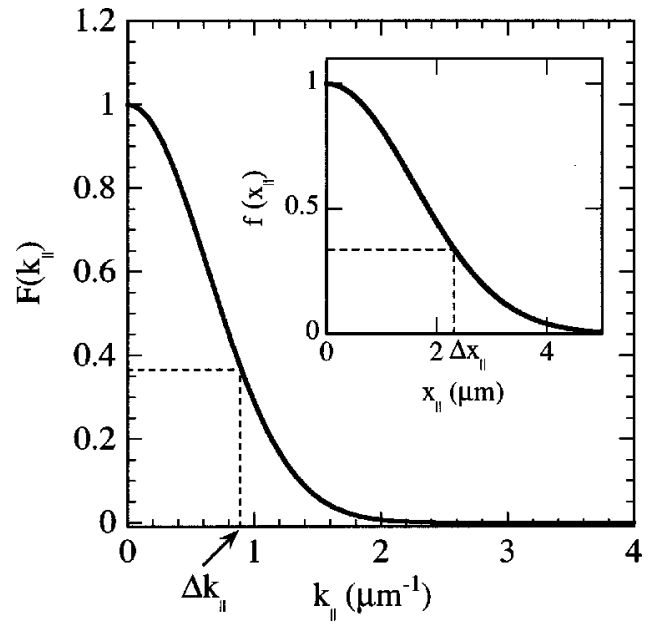

FIG. 1. Profile $F\left(k_{\|}\right)$of the initial lattice displacement in the wave-number space with $\Delta k_{\|}=(2 \pi / 7) \quad \mu \mathrm{m}^{-1}$. The inset shows the corresponding profile $f\left(x_{\|}\right)$in the real space with $\Delta x_{\|}=2 / \Delta k_{\|}$ $=7 / \pi \mu \mathrm{m}$.

separation of the grid points is $\Delta x=\Delta y=\Delta z=0.22 \mu \mathrm{m}$. These sizes of the time step and grid are determined from the convergence criteria of the FDTD scheme $\left(\Delta x>v_{x} \Delta t\right.$ with $v_{x}$ the sound velocity in the $x$ direction etc.). The width $\Delta x_{\|}=2 / \Delta k_{\|}=7 / \pi \mu \mathrm{m}$ for the displacement field is assumed for the Gaussian profile at $t=0$, as shown in Fig. 1 .
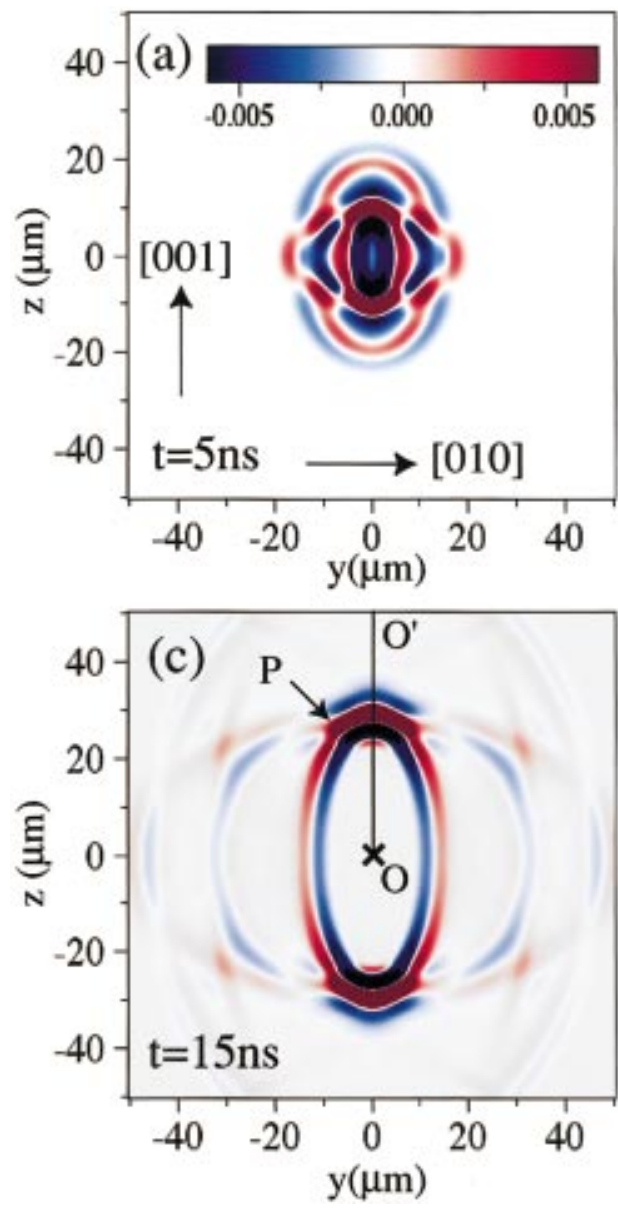
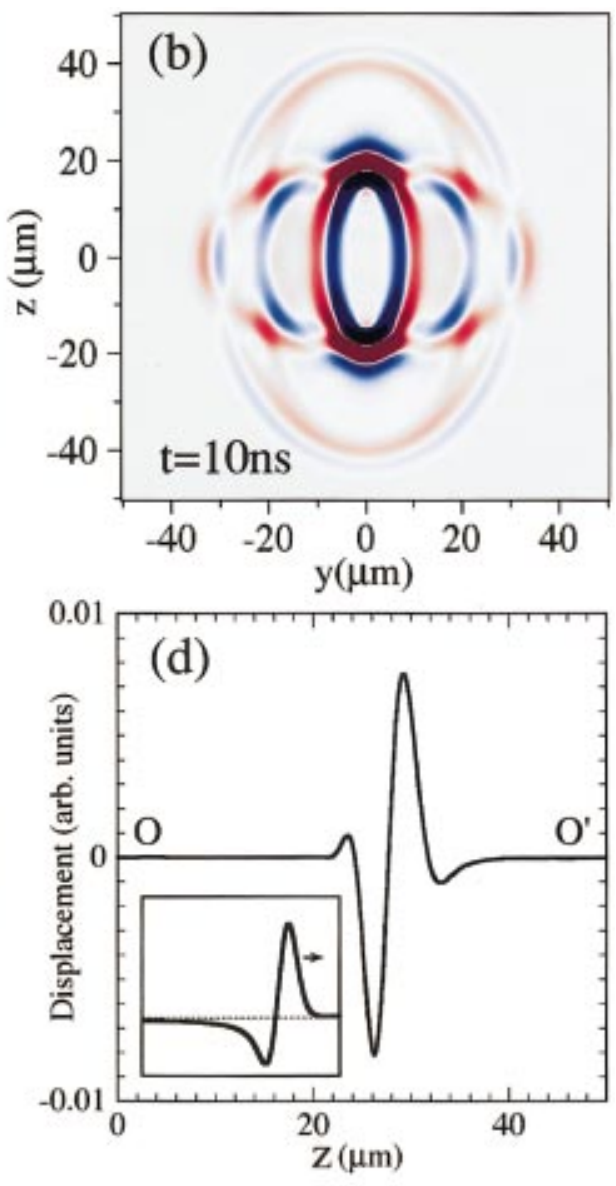

FIG. 2. (Color) (a)-(c) Displacement images $\left[u_{1}(x=0)\right.$ calculated by the FDTD method] for the (100) surface ( $Y Z$ plane) of $\mathrm{TeO}_{2}$ plotted at every $5 \mathrm{~ns}$ after excitation. The images span over $100 \mu \mathrm{m}$ both horizontally and vertically. (d) The displacement profile along the line connecting the points $O^{\prime}$ and $O$ shown in (c). The inset shows the profile calculated with Eq. (15). 


\section{A. Wave-front images in the (100) plane of $\mathrm{TeO}_{2}$}

Figures 2(a)-2(c) show the wave-front images (corresponding to the out-of-plane component $u_{1}$ that can be accessed experimentally) on the (100) plane at the three selected times $5 \mathrm{~ns}, 10 \mathrm{~ns}$, and $15 \mathrm{~ns}$, after the excitation of the waves. These theoretical images for the lattice displacement $u_{1}$ are consistent (both in shape and in brightness) with the experimental images for the particle velocity $\dot{u}_{1}$ obtained by Sugawara et $a .^{2}$ We see the dominant anisotropic signature of elliptical shape at the center together with faint signals outside. The latter disappears rather quickly as time elapses. In Fig. 2(d) we have illustrated the profiles of the wave amplitude along the $z$ axis (the [001] direction) at $t=15 \mathrm{~ns}$.

In order to identify the origins of these wave-front structures we have illustrated in Fig. 3(a) the group velocities of the bulk, surface $(S)$ and pseudosurface (PS1 and PS2) waves in the (100) plane overlapped with the theoretical wave-front image. To generate these plots for the surface (pseudosurface) mode, the directions of the wave vector $\mathbf{k}_{\|}$in the (100) plane are assumed to be distributed uniformly with the interval of $\Delta \theta_{\mathbf{k}_{\|}}=4^{\circ}\left(\Delta \theta_{\mathbf{k}_{\|}}=8^{\circ}\right)$. Thus the density of the points plotted represents the accumulation of the group-velocity vectors, reflecting phonon focusing effects ${ }^{18-22}$ at the surface. All these group-velocity curves coincide well with the locations of the wave fronts obtained by the FDTD calculations, though the structure of the PS1 (one of the pseudosurface wave branches) is not obvious in Figs. 2(a)-2(c) due to their polarizations (predominantly polarized parallel to the surface).

Now we see that the intense inner structure corresponds to the surface waves and the weak structures outside correspond to pseudosurface (PS2) and bulk waves. To understand the variation of the relative magnitudes of the wave amplitude appearing in the surface wave image, we also illustrate in Fig. 3(b) the density of the group-velocity vectors of the surface mode weighted by the square of the displacement amplitude $\left|u_{1}\right|^{2}$ at the surface $x=0$. The results reproduce semiquantitatively the relative magnitudes and their angular dependence shown in the FDTD images.

On the surface wave branch the displacement amplitude at the wave front becomes very large at $84^{\circ}$ rotated from the $[0 \overline{1} 0]$ direction [the region labeled $P$ in Fig. 2(c)]. The group velocities are accumulated closely in this direction as can be seen in Fig. 3(a). This is more evident in Fig. 4(a) where the pseudo-three-dimensional representation of the FDTD signal is displayed. Figure 4(b) shows a magnification of the group-velocity curve [equivalent to Fig. 3(a)] in the region marked $P$. We see that there exist two directions close together (the ones labeled $A$ and $B$ ) at which the groupvelocity curve folds. These directions are the caustics along which the wave intensity diverges in the geometrical acoustics approximation. The folding of the group-velocity curve induces a strong phonon focusing effect at the surface, which is quantitatively evaluated by the focusing factor $\mathcal{F}$ measuring the enhancement of acoustic flux relative to that in an isotropic medium. Explicitly, $\mathcal{F}$ is defined by $\mathcal{F}$ $=\left|\Delta \theta_{\mathbf{k}_{\|}} / \Delta \theta_{\mathbf{V}}\right|$, where $\Delta \theta_{\mathbf{k}_{\|}}$is a small angle occupied by the wave vector $\mathbf{k}_{\|}$in $2 \mathrm{D}$ wave-vector space and $\Delta \theta_{\mathbf{V}}$ is the

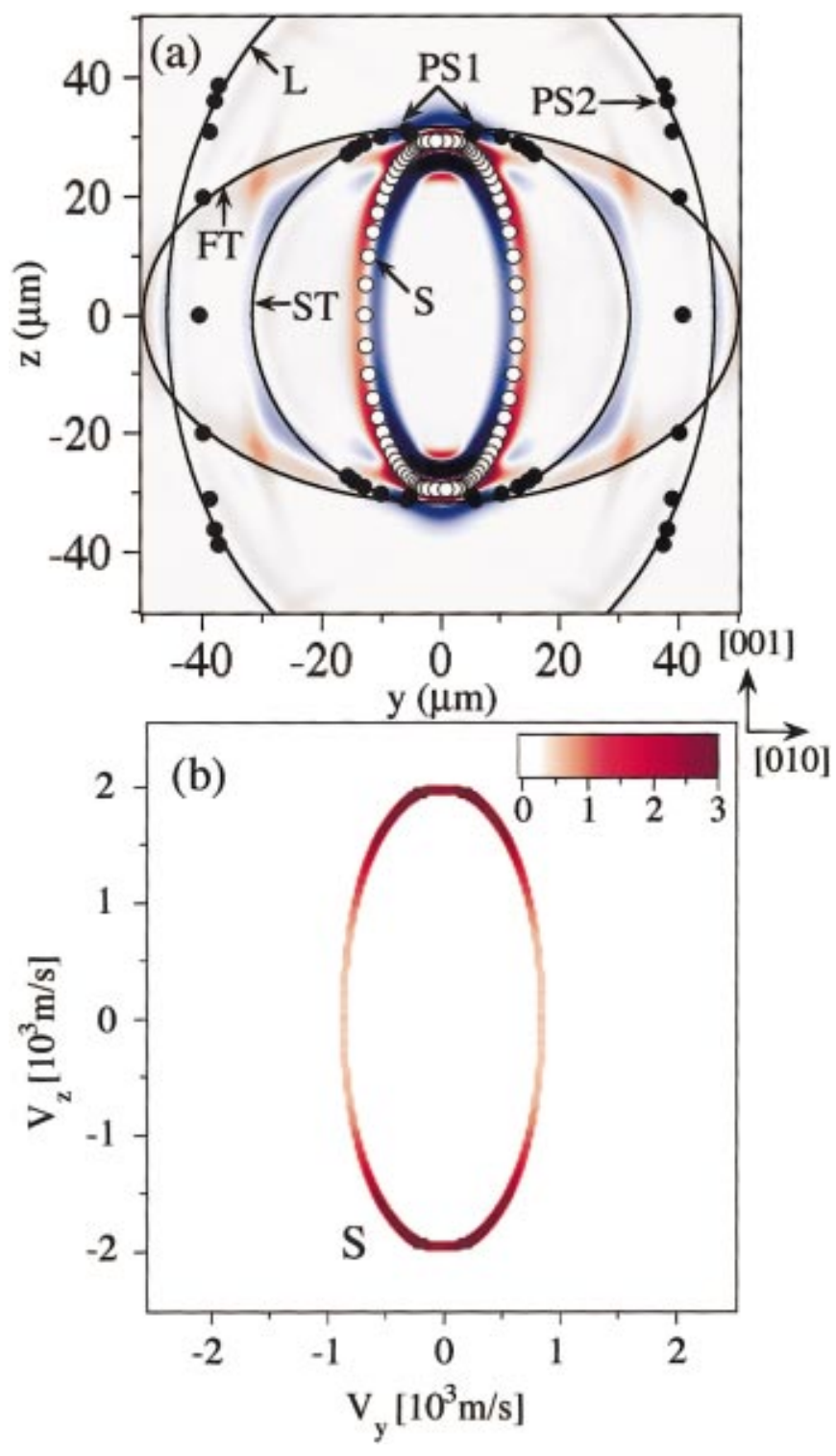

FIG. 3. (Color) (a) Distributions of the group velocities of the surface ( $S$, open circles), pseudosurface (PS1 and PS2, dots) and bulk acoustic waves [longitudinal $(L)$, slow-transverse (ST), and fast-transverse (FT), solid lines] overlapped with the wave-front image at $t=15 \mathrm{~ns}$. To generate this figure, the wave vectors are confined inside the (100) plane of $\mathrm{TeO}_{2}$ and they are assumed to be distributed uniformly. (b) Distributions of the group velocities of the surface mode $(S)$ weighted by the square of the out-of-surface component $\left|u_{1}(x=0)\right|^{2}$ of the lattice displacement at the surface. [The wave vectors are inside the (100) plane of $\mathrm{TeO}_{2}$.]

corresponding small angle subtended by the group-velocity vector $\mathbf{V}$ in the surface. In the calculation of $\mathcal{F}$ we have assumed a uniform distribution for the wave-vector directions of $\mathbf{k}_{\|}$. The resulting focusing factor $\mathcal{F}$ is shown in Fig. 4(c).

Here we remark on the profile of the lattice displacement versus distance in the surface (the FDTD result) shown in Fig. 2(d). In order to understand this numerical profile we solve the wave equation for a scalar displacement $u\left(x_{\|}, t\right)$ in an isotropic 2D space 


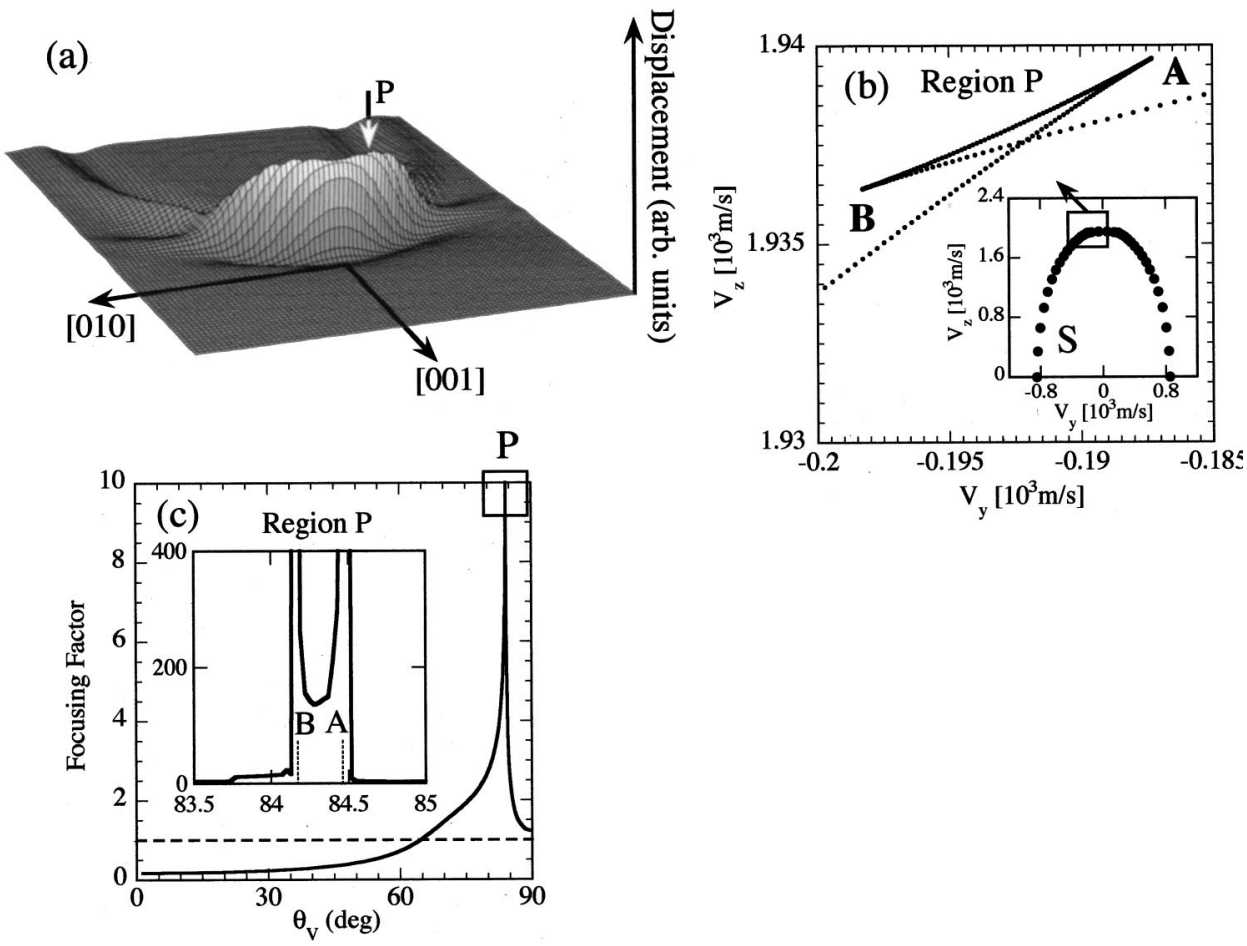

FIG. 4. (a) Pseudo-threedimensional representation of the displacement $u_{1}(x=0)$ perpendicular to the surface near the [001] direction. (b) Distribution of the group velocities in the region $P$ (rotated $\sim 84^{\circ}$ from the [010] direction). $A$ and $B$ are the caustics at which the group-velocity curve folds. In this figure the dots are used for indicating group velocities of the surface mode. (c) The phonon focusing factor versus group-velocity direction of the surface mode on the (100) surface (the [001] axis corresponding to $\theta_{V}=90^{\circ}$ ). Inset shows the magnification of the focusing factor in the region $P$.
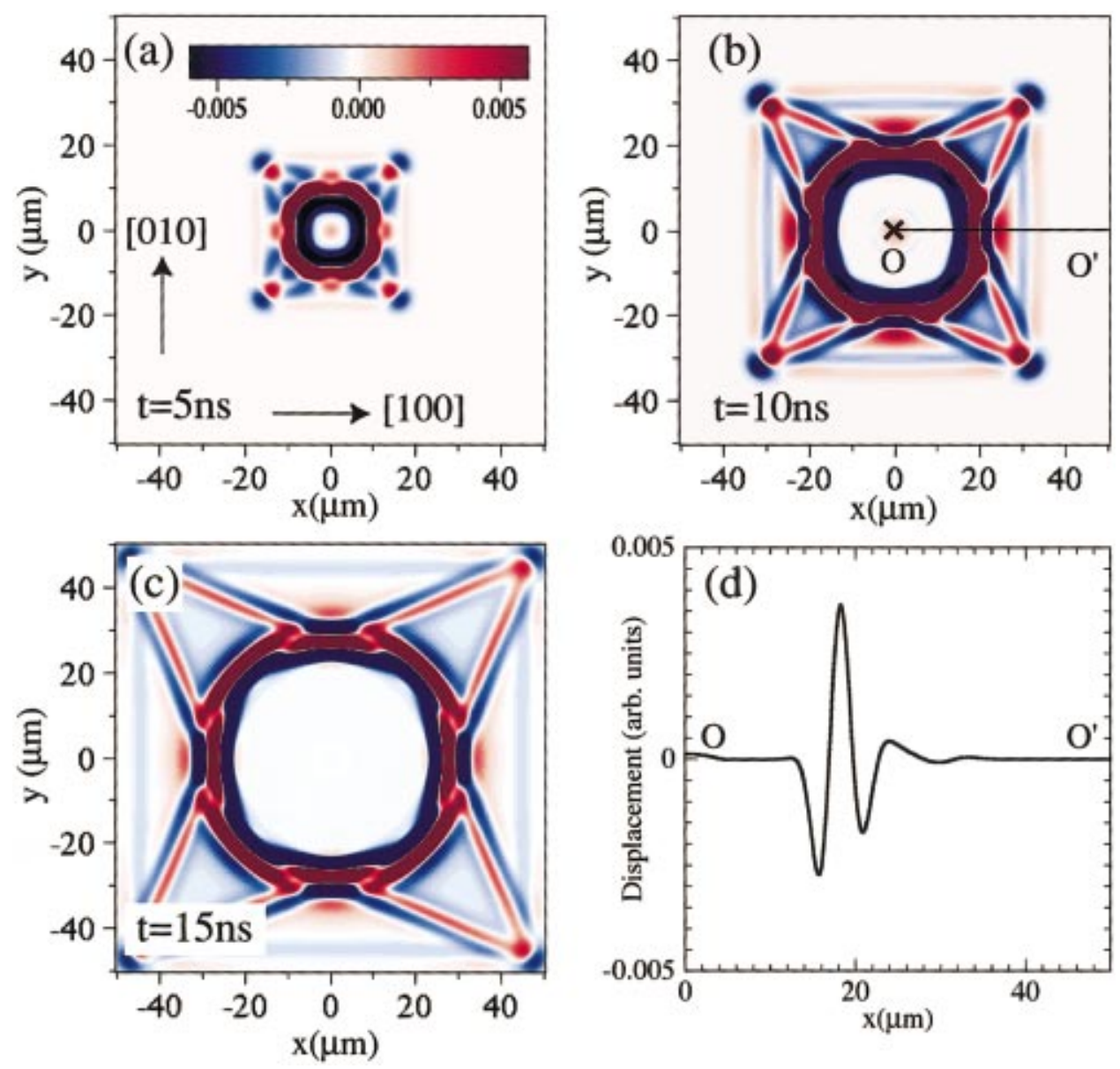

FIG. 5. (Color) (a)-(c) Displacement images $\left[u_{3}(z=0)\right.$ calculated by the FDTD method] on the (001) surface ( $X Y$ plane) of $\mathrm{TeO}_{2}$ plotted every 5 ns. The images span $100 \mu \mathrm{m}$ both horizontally and vertically. (d) The displacement profile along the line connecting the points $O^{\prime}$ and $O$ shown in (b). 


$$
\ddot{u}=v^{2}\left(\frac{\partial^{2} u}{\partial x_{\|}^{2}}+\frac{1}{x_{\|}} \frac{\partial u}{\partial x_{\|}}\right),
$$

under the initial conditions $u\left(x_{\|}, 0\right)=f\left(x_{\|}\right)$(with $f$ the Gaussian profile given in Sec. II D) and $\dot{u}\left(x_{\|}, 0\right)=0$. We find that the solution of Eq. (13) takes the form

$$
\begin{aligned}
u\left(x_{\|}, t\right)= & \int_{0}^{\infty} d k_{\|} k_{\|} J_{0}\left(k_{\|} x_{\|}\right) \cos \left[\omega\left(k_{\|}\right) t\right] \\
& \times \int_{0}^{\infty} d x_{\|}^{\prime} x_{\|}^{\prime} f\left(x_{\|}^{\prime}\right) J_{0}\left(k_{\|} x_{\|}^{\prime}\right) .
\end{aligned}
$$

The integral over $x_{\|}^{\prime}$ can be done analytically again with the Weber integral, Eq. (12), and we find

$$
\begin{aligned}
u\left(x_{\|}, t\right)= & \frac{2 \tilde{a}}{\left(\Delta k_{\|}\right)^{2}} \int_{0}^{\infty} k_{\|} J_{0}\left(k_{\|} x_{\|}\right) \\
& \times \exp \left(-\left[k_{\|} / \Delta k_{\|}\right]^{2}\right) \cos \left[\omega\left(k_{\|}\right) t\right] d k_{\|} .
\end{aligned}
$$

The integral over $k_{\|}$in Eq. (15) is carried out numerically. The calculated result for $u\left(x_{\|}, t\right)$ has been illustrated in the inset of Fig. 2(d), which reproduces semiquantitatively the oscillatory behavior of the displacement obtained by the FDTD calculation. A small discrepancy would be attributed to the fact that the initial disturbance is also converted to the bulk wave components and the medium supporting the surface acoustic waves is three dimensional.

\section{B. Wave-front images in the (001) plane of $\mathrm{TeO}_{2}$}

Similar but more interesting results for the (001) plane of $\mathrm{TeO}_{2}$ crystal are shown in Figs. 5(a)-5(c). A dramatic anisotropy in the wave-front image on this plane is the existence of the star-shaped structure exhibiting a four-fold symmetry outside the major round structure. Also a weak square structure surrounding the star-shaped structure is seen in Fig. 5(c), which is attributed to the bulk longitudinal waves. The central structure with large amplitude in the image is found to be woven from both the surface and pseudosurface waves. The profile of the displacement amplitude along the line connecting the points $O^{\prime}$ and $O$ in Fig. 5(d) exhibits more oscillations than those in the corresponding profile on the (100) plane shown in Fig. 2(d). This is because in the [100] direction (and its equivalent directions) on this surface, overlapping of the surface waves with different wave vectors exists due to the folded structure of the group-velocity curves, leading to the interference effects of the waves.

Figure 6(a) illustrates the group velocities of the bulk, surface, and pseudosurface waves in the (001) plane overlapped on the wave-front image. To generate this figure the directions of the wave vector $\mathbf{k}_{\|}$are again assumed to be distributed uniformly $\left(\Delta \theta_{\mathbf{k}_{\|}}=0.5^{\circ}\right)$ for the surface and pseudosurface modes. All these group-velocity curves coincide very well with the locations of the wave-fronts obtained by the FDTD calculations, though some structures of the group velocities do not have counterparts in the wave-front image. The latter group velocities are those of the waves that do not have the displacement component normal to the surface. We

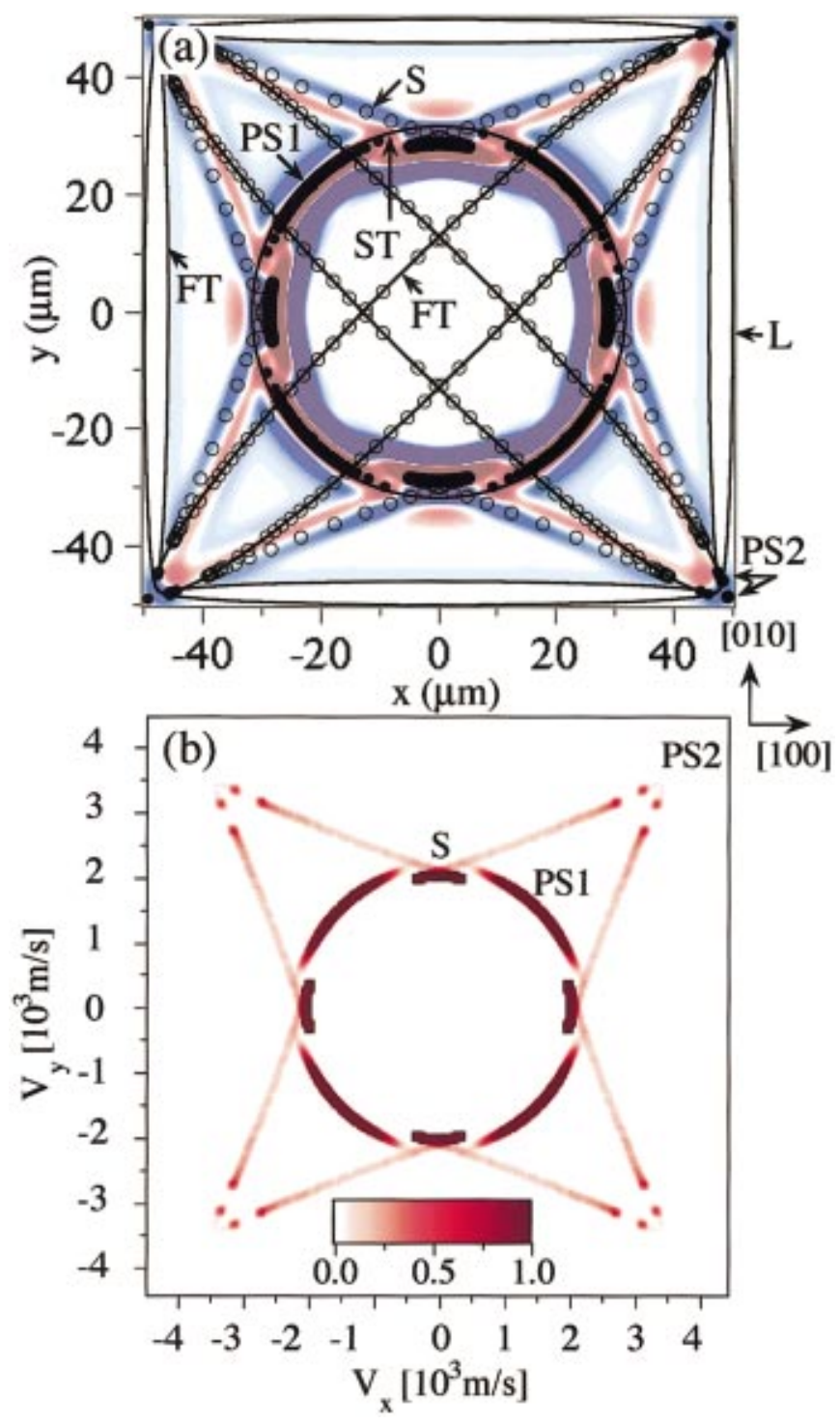

FIG. 6. (Color) (a) Distributions of the group velocities of the surface ( $S$, open circles), pseudosurface (PS1 and PS2, dots) and bulk acoustic waves [longitudinal $(L)$, slow-transverse (ST), and fast-transverse (FT), solid lines] overlapped with the wave-front image at $t=15 \mathrm{~ns}$. To generate this figure, the wave vectors are confined inside the (001) plane of $\mathrm{TeO}_{2}$ and they are assumed to be distributed uniformly (every $0.5^{\circ}$ in this plane). (b) Distributions of the group velocities weighted by the square of the out-of-plane component $\left|u_{3}(z=0)\right|^{2}$ of the lattice displacement at the surface $z$ $=0$. [The wave vectors are inside the (001) plane of $\mathrm{TeO}_{2}$ and both the surface $(S)$ and pseudosurface (PS1 and PS2) modes are displayed.]

have illustrated in Fig. 6(b) the density distribution of the group-velocity vectors of the surface and pseudosurface modes weighted by the square of the lattice displacement normal to the surface, i.e., $\left|u_{3}(z=0)\right|^{2}$. The results reproduce well the relative magnitude and the angular dependence shown in the wave-front images.

The PSW's are observed experimentally through the outof-plane component of displacement and the smallness of the magnitude of the attenuation coefficient $\varepsilon$ along the surface. 


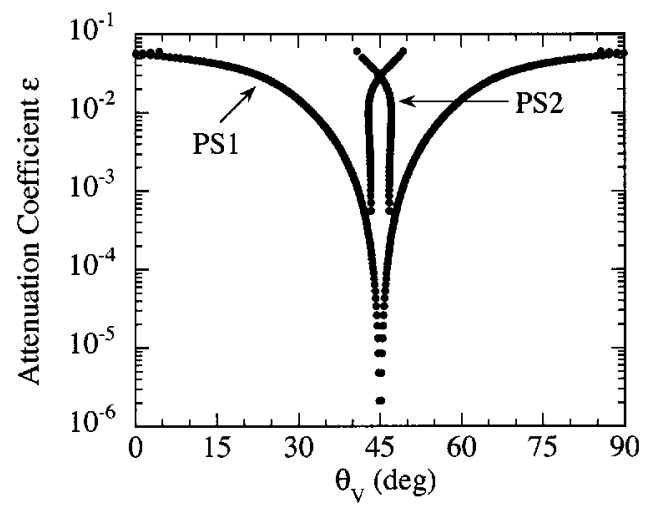

FIG. 7. Attenuation coefficient $\varepsilon$ versus propagation direction $\theta_{V}$ (the group-velocity direction measured from the [100] direction) of the pseudosurface waves propagating on the (001) surface of $\mathrm{TeO}_{2}$. PS1 and PS2 correspond to the pseudosurface branches shown in Fig. 6.

Figure 7 shows $\varepsilon$ for the PSW's on the (001) plane plotted as a function of group-velocity direction. The magnitude of the attenuation coefficient $\varepsilon$ is quite small, that is, it is typically $\sim 10^{-2}$ or less, indicating the small coupling to the bulk waves. Thus the PSW's can propagate along the surface almost unattenuated over 100 wavelengths $(\sim 1000 \mu \mathrm{m})$ or more. This is the reason why PSW's are observed experimentally just like true surface waves. ${ }^{23} \mathrm{We}$ also note that in the [110] direction $\left(\theta_{V}=45^{\circ}\right)$, one of the pseudosurface branches (labeled PS1) becomes a true surface mode without decay (i.e., $\varepsilon=0$ ) along the surface. Near this direction another branch of the PSW's (labeled PS2) appears.

\section{CONCLUDING REMARKS}

Stimulated by the recent imaging experiments by Wright and co-workers, ${ }^{2,3}$ we have studied theoretically the wavefront images of acoustic waves propagating on the (100) and (001) surfaces of a semi-infinite $\mathrm{TeO}_{2}$ crystal of tetragonal symmetry. The FDTD calculations reproduce very well the anisotropic features of the experimentally observed acoustic wave fronts arising from both surface and bulk propagations. However, the FDTD scheme does not directly give us information concerning the origin of those anisotropic structures. That can rather be provided by solving eigenvalue equations derived from the wave equations for the lattice displacements. With this latter method we could identify that the long-lived features of the wave fronts observed experimentally are composed of both surface and pseudosurface modes satisfying the stress-free boundary conditions at the surfaces and also having large displacement components perpendicular to the surfaces. Weak signals from bulk acoustic modes which do not satisfy the boundary conditions at the surface can also be seen, but they disappear rather quickly due to the decay into the bulk of the medium.

The FDTD method can also be applied to the calculation of the acoustic wave propagation in more complex surfaces than the homogeneous one studied here. An interesting example is a free or loaded surface with one- or twodimensional periodicity (i.e., surfaces of so-called phononic crystals) and also surfaces with roughness and other inhomogeneities. In our future studies we plan to extend the FDTD calculations to the acoustic wave propagation in such complex surfaces and interfaces.

\section{ACKNOWLEDGMENTS}

The authors would like to thank O. B. Wright for a critical reading of the manuscript. This work was supported in part by the Grant-in-Aid for Scientific Research from the Ministry of Education, Science and Culture of Japan (Grants No. 09640385, No. 12750002, and No. 14750045).

\section{APPENDIX}

In this appendix we give explicit expressions for the discretized versions of Eqs. (8) and (9).

$$
\begin{aligned}
& u_{1}^{i+1 / 2, j, k ; n+1}=2 u_{1}^{i+1 / 2, j, k ; n}-u_{1}^{i+1 / 2, j, k ; n-1} \\
& +\frac{(\Delta t)^{2}}{\rho}\left[\frac{\sigma_{11}^{i+1, j, k ; n}-\sigma_{11}^{i, j, k ; n}}{\Delta x}\right. \\
& +\frac{\sigma_{12}^{i+1 / 2, j+1 / 2, k ; n}-\sigma_{12}^{i+1 / 2, j-1 / 2, k ; n}}{\Delta y} \\
& \left.+\frac{\sigma_{13}^{i+1 / 2, j, k+1 / 2 ; n}-\sigma_{13}^{i+1 / 2, j, k-1 / 2 ; n}}{\Delta z}\right], \\
& u_{2}^{i, j+1 / 2, k ; n+1}=2 u_{2}^{i, j+1 / 2, k ; n}-u_{2}^{i, j+1 / 2, k ; n-1} \\
& +\frac{(\Delta t)^{2}}{\rho}\left[\frac{\sigma_{12}^{i+1 / 2, j+1 / 2, k ; n}-\sigma_{12}^{i-1 / 2, j+1 / 2, k ; n}}{\Delta x}\right. \\
& +\frac{\sigma_{22}^{i, j+1, k ; n}-\sigma_{22}^{i, j, k ; n}}{\Delta y} \\
& \left.+\frac{\sigma_{23}^{i, j+1 / 2, k+1 / 2 ; n}-\sigma_{23}^{i, j+1 / 2, k-1 / 2 ; n}}{\Delta z}\right], \\
& u_{3}^{i, j, k+1 / 2 ; n+1}=2 u_{3}^{i, j, k+1 / 2 ; n}-u_{3}^{i, j, k+1 / 2 ; n-1} \\
& +\frac{(\Delta t)^{2}}{\rho}\left[\frac{\sigma_{13}^{i+1 / 2, j, k+1 / 2 ; n}-\sigma_{13}^{i-1 / 2, j, k+1 / 2 ; n}}{\Delta x}\right. \\
& +\frac{\sigma_{23}^{i, j+1, k+1 / 2 ; n}-\sigma_{23}^{i, j-1 / 2, k+1 / 2 ; n}}{\Delta y} \\
& \left.+\frac{\sigma_{33}^{i, j, k+1 ; n}-\sigma_{33}^{i, j, k ; n}}{\Delta z}\right] \text {, } \\
& \sigma_{11}^{i, j, k ; n}=c_{11} \frac{u_{1}^{i+1 / 2, j, k ; n}-u_{1}^{i-1 / 2, j, k ; n}}{\Delta x} \\
& +c_{12} \frac{u_{2}^{i, j+1 / 2, k ; n}-u_{2}^{i, j-1 / 2, k ; n}}{\Delta y} \\
& +c_{13} \frac{u_{3}^{i, j, k+1 / 2 ; n}-u_{3}^{i, j, k-1 / 2 ; n}}{\Delta z},
\end{aligned}
$$




$$
\begin{aligned}
\sigma_{22}^{i, j, k ; n}= & c_{12} \frac{u_{1}^{i+1 / 2, j, k ; n}-u_{1}^{i-1 / 2, j, k ; n}}{\Delta x} \\
& +c_{11} \frac{u_{2}^{i, j+1 / 2, k ; n}-u_{2}^{i, j-1 / 2, k ; n}}{\Delta y} \\
& +c_{13} \frac{u_{3}^{i, j, k+1 / 2 ; n}-u_{3}^{i, j, k-1 / 2 ; n}}{\Delta z}, \\
\sigma_{33}^{i, j, k ; n}= & c_{13} \frac{u_{1}^{i+1 / 2, j, k ; n}-u_{1}^{i-1 / 2, j, k ; n}}{\Delta x} \\
& +c_{13} \frac{u_{2}^{i, j+1 / 2, k ; n}-u_{2}^{i, j-1 / 2, k ; n}}{\Delta y} \\
& +c_{33} \frac{u_{3}^{i, j, k+1 / 2 ; n}-u_{3}^{i, j, k-1 / 2 ; n}}{\Delta z}
\end{aligned}
$$

$$
\begin{aligned}
\sigma_{23}^{i, j+1 / 2, k+1 / 2 ; n}= & c_{44}\left[\frac{u_{2}^{i, j+1 / 2, k+1 ; n}-u_{2}^{i, j+1 / 2, k ; n}}{\Delta z}\right] \\
& \left.+\frac{\left.u_{3}^{i, j+1, k+1 / 2 ; n}-u_{3}^{i, j, k+1 / 2 ; n}\right]}{\Delta y}\right] \\
\sigma_{13}^{i+1 / 2, j, k+1 / 2 ; n}= & c_{44}\left[\frac{u_{1}^{i+1 / 2, j, k+1 ; n}-u_{1}^{i+1 / 2, j, k ; n}}{\Delta z}\right] \\
& \left.+\frac{u_{3}^{i+1, j, k+1 / 2 ; n}-u_{3}^{i, j, k+1 / 2 ; n}}{\Delta x}\right] \\
\sigma_{12}^{i+1 / 2, j+1 / 2, k ; n}= & c_{66}\left[\frac{u_{1}^{i+1 / 2, j+1, k ; n}-u_{1}^{i+1 / 2, j, k ; n}}{\Delta y}\right] \\
& \left.+\frac{\left.u_{2}^{i+1, j+1 / 2, k ; n}-u_{2}^{i, j+1 / 2, k ; n}\right]}{\Delta x}\right]
\end{aligned}
$$

where $(i, j, k)$ defines a three-dimensional grid point (grid point separations are $\Delta x, \Delta y$, and $\Delta z$ ) and $n$ specifies the time step with the interval $\Delta t$.
${ }^{1}$ B. A. Auld, Acoustic Fields and Waves in Solids (Wiley, New York, 1973), Vol. I.

${ }^{2}$ Y. Sugawara, O.B. Wright, O. Matsuda, M. Takigahira, Y. Tanaka, S. Tamura, and V.E. Gusev, Phys. Rev. Lett. 88, 185504 (2002).

${ }^{3}$ O.B. Wright, Y. Sugawara, O. Matsuda, M. Takigahira, Y. Tanaka, S. Tamura, and V.E. Gusev, Physica B 316-317, 29 (2002).

${ }^{4}$ C. Thomsen, H.T. Grahn, H.J. Maris, and J. Tauc, Phys. Rev. B 34, 4129 (1986).

${ }^{5}$ B. Taylor and H.J. Maris, Phys. Rev. Lett. 23, 416 (1969); Phys. Rev. B 3, 1462 (1971).

${ }^{6}$ G. A. Northrop and J. P. Wolfe, in Nonequilibrium Phonon Dynamics, edited by W. E. Bron (Plenum, New York, 1985), Chap. 5; J. P. Wolfe, Imaging Phonons (Cambridge University Press, Cambridge, UK, 1998).

${ }^{7}$ R.E. Camley and A.A. Maradudin, Phys. Rev. B 27, 1959 (1983).

${ }^{8}$ H.J. Maris, Phys. Rev. B 28, 7033 (1983).

${ }^{9}$ S. Tamura and M. Yagi, Phys. Rev. B 49, 17378 (1994).

${ }^{10}$ A. Taflove, Advances in Computational Electrodynamics: The Finite-Difference Time-Domain Method, edited by C. M. Soukoulis (Artech House, London, 1998).

${ }^{11}$ C.T. Chan, Q.L. Yu, and K.M. Ho, Phys. Rev. B 51, 16635 (1995).

${ }^{12}$ A.J. Ward and J.B. Pendry, Phys. Rev. B 58, 7252 (1998).
${ }^{13}$ M. Sigalas and N. Garcia, J. Appl. Phys. 87, 3122 (2000).

${ }^{14}$ Y. Tanaka, Y. Tomoyasu, and S. Tamura, Phys. Rev. B 62, 7387 (2000).

${ }^{15}$ T.C. Lim and G.W. Farnell, J. Acoust. Soc. Am. 45, 845 (1969).

${ }^{16}$ G. W. Farnell, in Physical Acoustics VI, edited by W. P. Mason and R. N. Thurston (Academic, New York, 1970), p. 109.

${ }^{17}$ For $\mathrm{TeO}_{2}$ we use the elastic constants $c_{11}=5.57, c_{33}=10.58$, $c_{12}=5.12, c_{13}=2.18, c_{33}=10.58, c_{44}=2.65$, and $c_{66}=6.59$ (in units of $10^{11} \mathrm{dyn} / \mathrm{cm}^{2}$ ) and mass density $\rho=5.99 \mathrm{~g} / \mathrm{cm}^{3}$.

${ }^{18}$ S. Tamura and K. Honjo, Jpn. J. Appl. Phys., Suppl. 20 3, 17 (1980).

${ }^{19}$ Al.A. Kolomenskii and A.A. Maznev, JETP Lett. 53, 423 (1991) [Pis'ma Zh. Eksp. Teor. Fiz. 53, 403 (1991)]; Phys. Rev. B 48, 14502 (1993).

${ }^{20}$ A.A. Maznev, Al.A. Kolomenskii, and P. Hess, Phys. Rev. Lett. 75, 3332 (1995).

${ }^{21}$ R.E. Vines, S. Tamura, and J.P. Wolfe, Phys. Rev. Lett. 74, 2729 (1995); 75, 1873(E) (1995).

${ }^{22}$ S. Tamura, R.E. Vines, and J.P. Wolfe, Phys. Rev. B 54, 5151 (1996).

${ }^{23}$ F.R. Rollins, Jr., T.C. Lim, and G.W. Farnell, Appl. Phys. Lett. 12, 236 (1968). 